[16] R. Sita Rama Chandra Rao and G. Sri Rama Chandra Murty, On Mirsky's generalisation of a problem of Evelyn-Linfoot and Page in additive theory of numbers, J. Reine Angew. Math. 309 (1979), 92-99.

[17] G. N. Watson, A treatise on the theory of Bessel functions, Cambridge University Press. 1966.

\section{On the greatest prime factor of $\prod_{k=1}^{x} f(k)$}

by

P. ERDös (Budapest) and A. Schinzel (Warszawa)

In memory of Trygue Nagell

Let $P(n)$ denote the greatest prime factor of $n$. T. Nagell was the first to give a non-trivial lower bound for $P\left(\prod_{k=1}^{x} f(k)\right)$, where $f$ is an arbitrary irreducible polynomial of degree greater than 1 . In [5] he proved

$$
P\left(\prod_{k=1}^{x} f(k)\right)>c(f, \varepsilon) x(\log x)^{1-\varepsilon} \quad \text { for all } \varepsilon>0 .
$$

In 1951 the first named author improved considerably the above inequality by proving that for $x>x_{0}(f)$

$$
P\left(\prod_{k=1}^{x} f(k)\right)>x(\log x)^{c(f) \log \log \log x} \quad \text { with } c(f)>0 .
$$

In the same paper [1] he has also claimed that

$$
P\left(\prod_{k=1}^{x} f(k)\right)>x \exp \left((\log x)^{\delta(s)}\right) \quad \text { with } \delta(f)>0 .
$$

Our efforts to reconstruct the proof of the latter estimate have been unsuccessful. Instead we have proved the following

THEOREM 1. Let $f \in \mathbf{Z}[x]$ be an irreducible polynomial of degree $l>1$. There exists an absolute constant $c_{1}>0$ such that for $x>x_{1}(f)$

$$
P\left(\prod_{k=1}^{x} f(k)\right)>x \exp \exp \left(c_{1}(\log \log x)^{1 / 3}\right) .
$$

In the sequel we shall denote the $n$th iterate of $\log x$ by $\log _{n} x$, the number of solutions of the congruence $f(k) \equiv 0(\bmod m)$ in the interval $1 \leqslant k \leqslant x$ by 
$\varrho_{x}(m)$, the number of divisors of an integer $n$ in a set $S$ by $d(n, S)$ and we shall put:

$$
\varrho_{m}(m)=\varrho(m) .
$$

$c_{1}, c_{2}, \ldots, x_{1}, x_{2}, \ldots$ will denote positive constants, in general depending on $f$, $p$ will denote primes.

Theorem 1 is an immediate consequence of the following two theorems.

THEOREM 2. Under the assumptions of Theorem 1 the number $N(x)$ of positive integers $k \leqslant x$ such that

$$
d\left(f(k),\left[\frac{x}{2}, x\right]\right) \geqslant 1
$$

satisfies for $x>x_{2}$

$$
N(x)>\frac{x}{\log x} \exp \left(c_{2}\left(\log _{2} x\right)^{1 / 3}\right),
$$

where $c_{2}$ is an absolute constant.

THEOREM 3. Under the assumptions of Theorems 1 and 2

$$
P\left(\prod_{k=1}^{x} f(k)\right)>x \exp \left(\frac{\log x}{l x} N(x)\right)
$$

for $x>x_{3}$.

The proof of Theorem 3 follows closely the proof of (1) given in [1]. It is clear from this theorem that in order to prove (2) it would be enough to show that

$$
N(x)>\frac{x}{(\log x)^{1-\delta(f)}} \quad \text { for } x>x_{4} .
$$

In this connection it is interesting to note that G. Tenenbaum [7] has obtained the asymptotic equality

$$
H_{f}(x, y, 2 y)=x /(\log y)^{1-\delta+o(1)}
$$

where the left-hand side is the number of positive integers $k \leqslant x$ such that $d(f(k),[y, 2 y]) \geqslant 1 ; x, y$ tend to infinity in the domain $y \leqslant x^{c_{0}}\left(c_{0}<1\right)$ and

$$
\delta=\frac{1+\log \log 2}{\log 2}
$$

Note added on April 27, 1989. Recently G. Tenenbaum has establish ed by a different method the inequality (2a), which implies (2) via Theorem ${ }^{3}$.

For the proof of Theorem 2 we require four lemmata.
LEMMA 1. If $x \geqslant m$ we have

$$
2 \frac{x}{m} \varrho(m) \geqslant \varrho_{x}(m) \geqslant \frac{1}{2} \frac{x}{m} \varrho(m) .
$$

Proof. We have for $x \geqslant m$

$$
\frac{x}{2 m} \varrho(m) \leqslant\left[\frac{x}{m}\right] \varrho(m) \leqslant \varrho_{x}(m) \leqslant\left[\frac{x}{m}\right] \varrho(m)+\varrho(m) \leqslant \frac{2 x}{m} \varrho(m) .
$$

LEMMA 2. If $z \geqslant 2 y, y>y_{1}$ we have

$$
2 \log \frac{\log z}{\log y} \geqslant \sum_{y \leqslant p \leqslant z} \frac{\varrho(p)}{p}>\frac{1}{2} \log \frac{\log z}{\log y} .
$$

Proof. We shall use the prime ideal theorem in the form

$$
\sum_{p \leqslant y} \varrho(p) \log p=y+O\left(y e^{-c_{3} \sqrt{\log y}}\right)
$$

(see [4], Satz 190).

By partial summation we obtain

hence

$$
\sum_{p \leqslant y} \frac{\varrho(p)}{p}=\log \log y+c_{4}+O\left(e^{-c_{3} \sqrt{\log y}}\right),
$$

$$
\sum_{y \leqslant p \leqslant z} \frac{\varrho(p)}{p}=\log \frac{\log z}{\log y}+O\left(e^{-c_{3} \sqrt{\log y}}\right)
$$

and since for $z \geqslant 2 y$ the main term dominates the error we get the desired bounds.

Lemma 3. Assume that $f$ is primitive. If $P$ runs through all int'sers composed of $n$ distinct prime factors we have for $y \geqslant y_{2}$

$$
\sum_{y / 4<P \leqslant y} \frac{\varrho(P)}{P}<\frac{c_{5} l^{n}\left(\log _{2} y+c_{6}\right)^{n-1}}{(n-1) ! \log y} .
$$

Proof. Since $\varrho(m)$ is multiplicative, we have $\varrho(P) \leqslant l^{n}$. On the other hand, for the number $\pi_{n}(x)$ of positive integers $\leqslant x$ composed of $n$ distinct prime factors we have the inequality (see [3])

$$
\pi_{n}(x) \leqslant \frac{c_{7} x\left(\log _{2} x+c_{6}\right)^{n-1}}{(n-1) ! \log x} .
$$

Hence

$$
\sum_{y / 4<P \leqslant y} \frac{1}{P}<\frac{4}{y} \pi_{n}(y) \leqslant \frac{c_{5}\left(\log _{2} y+c_{6}\right)^{n-1}}{(n-1) ! \log y} .
$$


Remark. The formulation of Lemma 3 and its proof have been corrected following a suggestion from $\mathrm{G}$. Tenenbaum.

LemMa 4. Let $c>0, r=\left[c\left(\log _{2} x\right)^{1 / 3}\right], A(c)$ be the set of all integers in the interval $[x / 2, x]$ of the form

$$
p q_{1} \cdots q_{r},
$$

where $p, q_{1}, \ldots, q_{r}$ are primes and for $i=1,2, \ldots, r$

(3) $\exp \left(\frac{1}{2(2 r+7)}(\log x)^{1-i / 2 r}\right)<q_{i}<\exp \left(\frac{1}{2(2 r+7)}(\log x)^{1-(i-1) / 2 r}\right)$.

The number $N_{0}(x)$ of positive integers $k \leqslant x$ such that

$$
d(f(k), A(c))>2 l r !(2 r+7)^{r+1}
$$

is $o\left(x /(\log x)^{r+2}\right)$.

Proof. We shall assume throughout that $x$ is sufficiently large and without loss of generality that $f$ is primitive. Then if $p q_{1} \cdots q_{r} \in[x / 2, x]$ and $q_{i}$ satisfy the inequalities (3) we have $p>x^{1 / 2}$. On the other hand for $k \leqslant x$

$$
|f(k)|<c_{8} x^{l},
$$

hence $f(k)$ can have at most $2 l$ prime factors greater than $x^{1 / 2}$. Therefore, (4) implies that $f(k)$ has more than

$$
R=r !(2 r+7)^{r+1}
$$

divisors in $A(c)$, of the form $p q_{1}^{(\sigma)} \cdots q_{r}^{(\sigma)}$, where $p$ is fixed.

Consider the family of sets $\left\{q_{1}^{(\sigma)}, \ldots, q_{r}^{(\sigma)}\right\}(1 \leqslant \sigma \leqslant R)$. By the theorem of Erdös and Rádo [2] the family contains a $\Delta$-system of cardinality $2 r+7$. Let the common intersection of any two distinct sets of this $\Delta$-system be $\left\{p_{1}, \ldots, p_{\delta}\right\}$, where $0 \leqslant \delta<r$. Let $s$ be the integer defined by

$$
2^{s-1}<\frac{x}{p p_{1} \cdots p_{\delta}} \leqslant 2^{s}
$$

By the condition $p q_{1}^{(\sigma)} \cdots q_{r}^{(o)} \in A(c) f(k)$ has at least $2 r+7$ pairwise coprime divisors in the interval $\left(2^{s-2}, 2^{5}\right]$, each divisor consisting of $r-\delta$ distinct prime factors all in the interval

$$
\left(\exp \left(\frac{1}{2(2 r+7)}(\log x)^{1 / 2}\right), \exp \left(\frac{1}{2(2 r+7)}(\log x)\right)\right)
$$

and all but one less than

$$
\exp \left(\frac{1}{2(2 r+7)}(\log x)^{1-1 / 2 r}\right)
$$

Hence

and

$$
s \geqslant \frac{(\log x)^{1 / 2}}{2(2 r+7) \log 2}=s_{0}
$$

$$
N_{0}(x) \leqslant \sum_{s=0}^{r-1} \sum_{s \geqslant s_{0}} \sum^{*} \varrho_{x}\left(P_{1} P_{2} \cdots P_{2 r+7}\right)
$$

Where the sum $\sum^{*}$ is taken over all sets of $2 r+7$ pairwise coprime integers $P_{1}, P_{2}, \ldots, P_{2 r+7}$ in the interval $\left(2^{s-2}, 2^{s}\right]$, each integer consisting of $r-\delta$ distinct prime factors of the size described above. For every such set we have

$$
P_{1} \cdots P_{2 r+7}<x^{1 / 2} \exp \left(\frac{r-\delta-1}{2}(\log x)^{1-1 / 2 r}\right)<x,
$$

thus by Lemma 1

$$
\varrho_{x}\left(P_{1} \cdots P_{2 r+7}\right)<2 \frac{\varrho\left(P_{1} \cdots P_{2 r+7}\right)}{P_{1} \cdots P_{2 r+7}} x=2 x \prod_{v=1}^{2 r+7} \frac{\varrho\left(P_{v}\right)}{P_{v}}
$$

and by (6)

$$
N_{0}(x) \leqslant \sum_{\delta=0}^{r-1} \sum_{s \geqslant s_{0}} \sum^{*} 2 x \prod_{v=1}^{2 r+7} \frac{\varrho\left(P_{v}\right)}{P_{v}} \leqslant 2 x \sum_{\delta=0}^{r-1} \sum_{s \geqslant s_{0}}\left(\sum \frac{\varrho(P)}{P}\right)^{2 r+7},
$$

Where $P$ runs through all integers $P$ in the interval $\left(2^{s-2}, 2^{5}\right]$ consisting of $r-\delta$ distinct prime factors. By Lemma 3 we obtain

$$
\sum \frac{\varrho(P)}{P} \leqslant \frac{c_{5} l^{r-\delta}\left(\log \log 2^{s}+c_{6}\right)^{r-\delta-1}}{(r-\delta-1) ! \log 2^{s}} \leqslant \frac{c_{9} l^{r-\delta}(\log s)^{r-\delta-1}}{(r-\delta-1) ! s}
$$

hence

(7) $\quad N_{0}(x) \leqslant 2 x \sum_{\delta=0}^{r-1}\left(\frac{c_{9} l^{r-\delta}}{(r-\delta-1) !}\right)^{2 r+7} \sum_{s \geqslant s_{0}} \frac{(\log s)^{(r-1)(2 r+7)}}{s^{2 r+7}}$.

For $s>s_{0}-1$ we have

$$
\log s>\log \left(s_{0}-1\right)>\frac{2 r+7}{2 r+5}(r-1)>r-1 .
$$

Therefore, on this halfine $(\log s)^{(r-1)(2 r+7) / s^{2 r+7}}$ is decreasing, since $\log s>r-1$ and

$$
\frac{(\log s)^{(r-1)(2 r+7)}}{s^{2 r+7}}<\frac{d}{d s}\left(-\frac{(\log s)^{(r-1)(2 r+7)}}{s^{2 r+6}}\right)
$$

since

$$
\log s>\frac{2 r+7}{2 r+5}(r-1)
$$


It follows that

$$
\begin{aligned}
\sum_{s \geqslant s_{0}} \frac{(\log s)^{(r-1)(2 r+7)}}{s^{2 r+7}} & \leqslant \int_{s_{0}-1}^{\infty} \frac{(\log s)^{(r-1)(2 r+7)}}{s^{2 r+7}} d s \\
& \leqslant \frac{\left(\log \left(s_{0}-1\right)\right)^{(r-1)(2 r+7)}}{\left(s_{0}-1\right)^{2 r+6}}=\frac{\exp O\left(r^{2} \log _{3} x\right)}{(\log x)^{r+3}}
\end{aligned}
$$

and by (7)

$$
N_{0}(x) \leqslant 2 x\left(c_{9} l e^{l}\right)^{2 r+7} \frac{\exp O\left(r^{2} \log _{3} x\right)}{(\log x)^{r+3}}=o\left(\frac{x}{(\log x)^{r+2}}\right) .
$$

Proof of Theorem 2 . For $k \leqslant x$ by (5) $f(k)$ has less than $c_{10} \log x$ prime factors. Thus we have in the notation of Lemma 4

$$
d(f(k), A(c))<\left(\begin{array}{c}
c_{10} \log x \\
r+1
\end{array}\right)<\frac{c_{10}^{r+1}}{(r+1) !}(\log x)^{r+1}
$$

From Lemma 4 and (8) we obtain

$$
\Sigma^{+} d(f(k), A(c))=o\left(\frac{x}{\log x}\right)
$$

where in $\sum^{+} k$ runs through all positive integers $k \leqslant x$ with

$$
d(f(k), A(c))>2 l r !(2 r+7)^{r+1} .
$$

On the other hand, by Lemma 1

$$
\sum_{k=1}^{x} d(f(k), A(c))=\sum_{a \in A(c)} \varrho_{x}(a) \geqslant \frac{x}{2} \sum_{a \in A(c)} \frac{\varrho(a)}{a} .
$$

We evidently have

$$
\sum_{a \in A(c)} \frac{\varrho(a)}{a}=\sum_{1} \frac{\varrho\left(q_{1}\right)}{q_{1}} \sum_{2} \frac{\varrho\left(q_{2}\right)}{q_{2}} \cdots \sum_{r} \frac{\varrho\left(q_{r}\right)}{q_{r}} \sum_{r+1} \frac{\varrho(p)}{p},
$$

where the sum $\sum_{i}$ is taken over all primes $q_{i}$ in the interval $(3)(1 \leqslant i \leqslant r)$ and the sum $\sum_{r+1}$ is taken over all primes $p$ in the interval

$$
\frac{x}{2 q_{1} \cdots q_{r}} \leqslant p \leqslant \frac{x}{q_{1} \cdots q_{r}} .
$$

It follows from Lemma 2 that

$$
\begin{gathered}
\sum_{i} \frac{\varrho\left(q_{i}\right)}{q_{i}}>\frac{1}{4 r} \log _{2} x \quad(1 \leqslant i \leqslant r), \\
\sum_{r+1} \frac{\varrho(p)}{p}>\frac{1}{2} \log \left(1+\frac{\log 2}{\log \left(x / 2 q_{1} \cdots q_{r}\right)}\right)>\frac{\log 2}{2 \log x} .
\end{gathered}
$$

Therefore,

$$
\sum_{a \in A(c)} \frac{\varrho(a)}{a}>\left(\frac{\log _{2} x}{4 r}\right)^{r} \frac{\log 2}{2 \log x}
$$

and by (10)

$$
\sum_{k=1}^{x} d(f(k), A(c))>\frac{\log 2}{4} \frac{x}{\log x}\left(\frac{\log _{2} x}{4 r}\right)^{r} .
$$

Since $r=o\left(\log _{2} x\right)$, it follows from (9) that

$$
\Sigma^{-} d(f(k), A(c))>\frac{x}{6 \log x}\left(\frac{\log _{2} x}{4 r}\right)^{r},
$$

where $\Sigma^{-}$is taken over all positive integers $k \leqslant x$ such that

$$
d(f(k), A(c)) \leqslant 2 l r !(2 r+7)^{r+1}
$$

From (11) we obtain

$$
\begin{aligned}
N(x) & >\frac{1}{12 \operatorname{lr} !(2 r+7)^{r+1}} \frac{x}{\log x}\left(\frac{\log _{2} x}{4 r}\right)^{r} \\
& >\frac{x}{\log x} \exp \left(r\left(\log _{3} x-3 \log r+1-\log 8+O\left(\frac{\log r}{r}\right)\right)\right) \\
& >\frac{x}{\log x} \exp \left(c\left(\log _{2} x\right)^{1 / 3}(-3 \log c+1-\log 8)+O\left(\log _{3} x\right)\right) .
\end{aligned}
$$

Choosing $c<\sqrt[3]{e / 8}$ (the choice $c=\sqrt[3]{1 /\left(8 e^{2}\right)}$ is optimal) we obtain the theorem.

Remark. If instead of the theorem of Erdös and Rádo we use their conjecture $r !(2 r+7)^{r+1}$ is replaced throughout by $(2 r+7)^{r+1}$ and the above proof for $r=\left[c^{\prime}\left(\frac{\log _{2} x}{\log _{3} x}\right)^{1 / 2}\right]$ gives

$$
N(x)>\frac{x}{\log x} \exp \left(c_{11}\left(\frac{\log _{2} x}{\log _{3} x}\right)^{1 / 2} \log _{4} x\right) \text { for } x>x_{4},
$$

Where $c_{11}$ is an absolute constant.

We proceed to the proof of Theorem 3. Denote by $U$ the set of all integers $u$ of the interval $(x / \log x, x]$ for which $f(u)$ has no prime factor satisfying

$$
x<p \leqslant c_{12} x, \quad \text { where } c_{12}^{l-1}=2 c_{8} \text {. }
$$

LEMMA 5. card $U>x-c_{13} \frac{x}{\log x}$. 
Proof. Clearly

$$
\begin{aligned}
\operatorname{card} U & =[x]-\left[\frac{x}{\log x}\right]-\sum_{x<p \leqslant c_{12} x}\left(\varrho_{x}(p)-\varrho_{\frac{x}{\log x}}(p)\right) \\
& >x-\frac{x}{\log x}-1-\operatorname{l\pi }\left(c_{12} x\right)>x-c_{13} \frac{x}{\log x} .
\end{aligned}
$$

For $k \leqslant x$ put

$$
|f(k)|=A_{k} B_{k}, \quad \text { where } A_{k}=\prod_{\substack{p^{\alpha} \| f(k) \\ p \leq x}} p^{\alpha}, B_{k}=|f(k)| / A_{k}
$$

and let

$$
P\left(\prod_{k=1}^{x} f(k)\right)=P_{x} .
$$

LemMa 6. For all $u \in U$

$$
A_{k}>\frac{x^{l}}{2(\log x)^{l} P_{x}^{l-1}} .
$$

Proof. Since by the definition of $U: x / \log x<u \leqslant x$ we have for $x>x$,

$$
\frac{1}{2}\left(\frac{x}{\log x}\right)^{\prime}<|f(u)|<c_{8} x^{l} .
$$

Further, $f(u)$ has no prime factor in the interval $\left(x, c_{12} x\right]$. Therefore by (12) and the choice of $c_{12} B_{u}$ can have at most $l-1$ prime factors, multiple factors counted multiply. By (13) all prime factors of $f(u)$ are at most $P_{x}$, thus

$$
B_{u} \leqslant P_{x}^{l-1} \text {. }
$$

Hence

$$
A_{u}=\frac{|f(u)|}{B_{u}}>\frac{x^{l}}{2(\log x)^{l} P_{x}^{l-1}} .
$$

Lemma 7. Let $u \in U$ be such that $f(u)$ has a divisor in $[x / 2, x]$. Then

$$
A_{u}>\frac{x^{l},}{2(\log x)^{l} P_{x}^{l-2}} .
$$

Proof. By the definition of $U$ all prime factors of $B_{u}$ are greater than $c_{12} x$. Since $f(u) \equiv 0(\bmod d)$ for some $d \in[x / 2, x]$ we have by $(12),(14)$ and the choice of $c_{12}$

$$
B_{u}<2 c_{8} x^{t-1}=\left(c_{12} x\right)^{t-1} .
$$

Thus $B_{u}$ can have at most $l-2$ prime factors, multiple factors counted multiply. Thus by (12) and (13)

$$
A_{u}=\frac{|f(u)|}{B_{u}}>\frac{x^{l}}{2(\log x)^{l} P_{x}^{l-2}} .
$$

\section{Lemma 8.}

$$
\sum_{k=1}^{x} \log A_{k}<x \log x+c_{14} x .
$$

Proof, see Nagell [6], pp. 180-182.

Proof of Theorem 3. The number of $u \in U$ for which $f(u)$ has a divisor in $[x / 2, x]$ is at least equal to $N(x)-(x-\operatorname{card} U)$, hence by Lemma 5 is at least $N(x)-c_{9} x / \log x$. From Lemmata $5,6,7$, and (8) we now obtain

$x \log x+c_{14} x$

$$
\begin{array}{r}
\geqslant \sum_{u \in U} \log A_{u}>\left(x-c_{13} \frac{x}{\log x}\right)\left(l \log x-l \log _{2} x-(l-1) \log P_{x}-\log 2\right) \\
+\left(N(x)-c_{13} \frac{x}{\log x}\right) \log P_{x}
\end{array}
$$

$>l x \log x-l x \log _{2} x-(l-1) x \log P_{x}-x-c_{13} l x$

$$
+c_{13}(l-1) \frac{x}{\log x} \log P_{x}+N(x) \log P_{x}-c_{13} \frac{x}{\log x} \log P_{x}
$$

$>l x \log x-l x \log _{2} x-(l-1) x \log P_{x}-\left(c_{13} l+1\right) x+N(x) \log P_{x}$. Hence

(15) $\quad(l-1) x \log \frac{P_{x}}{x}>N(x) \log P_{x}-l x \log _{2} x-\left(c_{13} l+c_{14}+1\right) x$.

By Lemma 2 for $x>x_{6}$ there is at least one prime $p \in[x / 2, x]$ with $\varrho(p)>0$, hence $P_{x} \geqslant x / 2$. On the other hand, by Theorem $1 x \log _{2} x=o(N(x) \log x)$. Thus for $x>x_{7}$

$$
l x \log _{2} x+\left(c_{13} l+c_{14}+1\right) x<\frac{1}{l} N(x) \log x-N(x) \log 2
$$

and the inequality (15) gives

i.e.

$$
\begin{gathered}
(l-1) x \log \frac{P_{x}}{x}>\frac{l-1}{l} N(x) \log x, \\
P_{x}>x \exp \left(\frac{\log x}{l x} N(x)\right),
\end{gathered}
$$

Which was to be proved. 


\section{References}

[1] P. Erdös, On the greatest prime factor of $\prod_{k=1}^{x} f(k)$, J. London Math. Soc. 27 (1952), 379-384.

[2] P. Erdös and R. Rảdo, Intersection theorems for systems of sets, ibid. 35 (1960), 85-90.

[3] G. H. Hardy and S. Ramanujan, The normal number of prime factors of a number ${ }^{m}$ Quarterly J. Math. 48 (1917), 76-92.

[4] E. Landau, Einführung in die elementare und analytische Theorie der algebraischen Zahlen und der Ideale, Chelsea 1949.

[5] T. Nagell, Généralisation d'un théorème de Tchebycheff. J. Math. Pur. Appl. (8) 4 (1921) 343-356.

[6] -Zur Arithmetik der Polynome, Abh. Math. Seminar Univ. Hamburg 1 (1922), 179-194.

[7] G. Tenen ba u m, Sur une question d'Erdös et Schinzel, the volume: A tribute to Paul Erdös to be published by the Cambridge University Press.

Received on 21.2.1989

and in revised form on 28.4.1989 